\title{
Correspondence
}

To the Editors

\section{Guidelines for the use of surfactant in Sri Lanka}

\author{
Sri Lanka Journal of Child Health, 2008; 37: 103-104
}

Surfactant was made available through the Medical Supplies Division (MSD) to the National Health Service (NHS) for the use of paediatricians / neonatalogists in the treatment of neonatal respiratory distress syndrome due to surfactant deficiency. Its cost is approximately Rs. 25,000 per vial. The Perinatal Society of Sri Lanka (PSSL) has been working hard to get this for the last 6 years.

Surfactant is a complex mixture of phospholipids, neutral lipids and proteins produced by type II alveolar cells which become prominent in fetal lung after 24 weeks of gestation ${ }^{1}$. Granules of surfactant are stored in the lamellar bodies of type II cells and secreted into the alveoli ${ }^{1}$. Surfactant reduces the surface tension of alveoli by becoming a solid monolayer promoting stability of alveoli thus preventing atelectasis and reducing the work of breathing ${ }^{1}$. These surface properties result from its composition. The major constituents of surfactant are lipids the most important of which are phosphatidylcholine (PC) and phosphatidylglycerol (PG) comprising $70-80 \%$ and $5-10 \%$ of the lipids, respectively ${ }^{1}$. Another $10 \%$ of the lipids is made up of phosphatidylinositol (PI), phosphatidyl serine (PS) and phosphatidylethanolamine (PE) ${ }^{1}$. Ten percent are neutral lipids such as cholesterol and sphingomyelin ${ }^{1}$. Surfactant proteins (SP) are called A, B, C and $\mathrm{D}^{1}$. Lipids, especially dipalmitoyl phosphatidylcholine (DPPC), are the most important component for surface activity of surfactant ${ }^{1}$. The concentration of PC increases rapidly in the fetal lung towards term. Surfactant proteins, especially SP-B and SP-C, are important contributors towards surface activity ${ }^{1}$. SPA and SP-D have other roles. Ninety percent of native and exogenously administered surfactant is recycled in the lung ${ }^{1}$.

Neonatal respiratory distress syndrome associated with prematurity related surfactant deficiency was first described in 1950s. Surfactant was introduced as an investigational new drug for treatment of this condition in late 1980s. Both artificial and natural surfactants were used under research settings until early 1990s. Surfactant administration became a routine practice in early $1990^{2}$. It remains an expensive drug even today.
There is clear evidence of surfactant being beneficial in the treatment of RDS of prematurity. The benefits include reduction in ventilatory requirements, oxygen requirements and the mortality related to $\mathrm{RDS}^{2}$. There is a reduction in the incidence of pneumothoraces, pneumomediastinum, pneumopericardium and pulmonary interstitial emphysema with the use of surfactant ${ }^{2}$. Effects are unclear on intraventricular hemorrhage, bronchopulmonary dysplasia patent ductus arteriosus and necrotizing enterocolitis ${ }^{2}$.

Natural surfactant is superior to artificial surfactant in clinical trials due to presence of SP-B ${ }^{3}$. Prophylactic surfactant to high risk groups (e.g. $500-900 \mathrm{~g}$ or 24 to 31 weeks) is better and more effective than rescue surfactant therapy once respiratory distress has developed ${ }^{3}$. Early rescue surfactant ( 2 or more hours after birth) is better than late rescue (after 6 hours) surfactant for $\mathrm{RDS}^{3}$.

The neonatal mortality reduced from $9.7 / 1000$ live births to $9.1 / 1000$ live births (6\% reduction) purely due to surfactant usage in USA from 1989 to $1990^{2}$. This mortality reduction is mainly from survival of 500 to $1250 \mathrm{~g}$ birth weight infants and not from the survival of 1250 to $1500 \mathrm{~g}$ birth weight infants ${ }^{2}$. Antenatal steroids have additive effects on surfactant ${ }^{1}$. Surfactant has been shown to be beneficial in other conditions such as meconium aspiration syndrome (MAS) and asphyxial lung disease when given in large doses and multiple doses $^{3}$.

In this background when surfactant was introduced to NHS Sri Lanka the PSSL took the initiative to issue guidelines for its use. The guidelines of the PSSL were formulated at the advanced neonatal ventilation workshop held in April 2008. There were over 64 paediatricians in the workshop with 6 expert neonatologists from India who contributed to the decision making process. Therefore it was a valid forum to make decisions and communicate it effectively to get consensus for implementation. The guidelines were as follows. 
Surfactant should be used in neonatal intensive care units for the treatment of radiologically confirmed neonatal RDS due to surfactant deficiency.(rescue therapy as early as possible). It should not be used for prophylaxis of this condition in high risk categories or for any other indication such as MAS. It should be used selectively for neonatal RDS for infants

- $\quad$ with a birth weight above 900 grams

- with a maturity above 27 weeks gestation.

- in the absence major congenital abnormalities.

- $\quad$ in the absence of asphyxia.

It is not legally binding to adhere to guidelines at the moment. Our justification for guidelines is as follows: One hundred vials of surfactant have been issued for the entire country through MSD. In general, incidence of prematurity is $10 \%$ of total births $(32,500$ of 325,000 births in Sri Lanka). If $1 \%$ of them develop neonatal RDS 3,250 babies would develop it annually. We suggested providing surfactant for $10 \%$ of these babies i.e. 300 vials of surfactant for the first year. Ministry of Health provided us with 100 vials of surfactant for this year. We are happy about it as this is a good start. We should be accountable for its use. We should be able to save at least 80 lives with these 100 vials. Therefore we suggested to the MSD and FHB to monitor its use so that it is not misused and wanted the MSD to issue a circular on this. This may happen in the future. We also should monitor both the short term and the long term outcome after its use. Therefore a format was prepared and submitted to the MSD to annex with the circular. This should be filled and returned to the FHB.

We discussed the pros and cons of these guidelines. There should be certain facilities to manage infants who receive surfactant. One should have facilities to ventilate such infants, if required, following administration of surfactant. Hence it's use should be limited to NICUs. The amount of surfactant available is inadequate to administer it prophylactically. We do save babies below $900 \mathrm{~g}$ of birth weight and less than 27 weeks of gestation in our units. However, we do not have data on these or even the data from neonatal units in our own country. The facilities required and support needed to save this group of infants are not widely available. Surfactant is not the only requirement for these infants. We do not have data on mortality of each neonatal unit. If a unit has a NMR of $40-50 \%$ of admissions then that unit has deaths due to many causes other than non-availability of surfactant. Therefore, before making surfactant available, our neonatal units must look at their own mortality, conduct audit meetings and look at care issues contributing towards that mortality such as cross infections, hand washing practices, ventilation strategies and staff training in ventilation strategies for middle grade medical officers (quality issues). More importantly we should develop a Perinatal Information Network in a National Perinatal Information Centre to collect all the required information to make such policies before requesting the already stretched national budget to fund surfactant in the NHS on a wider scale. Definitely surfactant is effective in reducing mortality in infants of 500-999g s but we need to know the numbers first to cost it. The same arguments are against asphyxia and major congenital abnormalities. The drug is not freely available and it will never be. There is an ethical issue (resource ethics) surrounding its use. If you had known that the infant with RDS has major congenital abnormalities and was asphyxiated at birth restrict its use. Of course still the decision making right is with the paediatrician and they can be guided by these guidelines. Ultimately if we could use it carefully and save lives it will help us to get more surfactant into the NHS with supporting evidence on hard data. We will continue with this dialogue in the future on surfactant and help develop perinatal services in the country over the next decade. If we can contribute to a reduction of NMR by $0.6 / 1000$ live births with surfactant as in $\mathrm{USA}^{2}$ our NMR can be reduced to a single digit figure in 2 years along with the on going NALS training. That is one fifth of Millennium Development Goal 4.

\section{References}

1. Rennie JM, editor Roberton Textbook of Neonatology. $4^{\text {th }}$ ed. London; Churchill Livingstone 2005. p. 456-63.

2. Rochel M, Schwartz RM, Luby AM, Scanlon JW, Kellogg RJ. Effects of surfactant on morbidity, mortality and resource use in newborn infants weighing 500 to $1500 \mathrm{~g}$. The New England Journal of Medicine 1994; 330: 1476-80.

3. Engle WA. Surfactant-Replacement therapy for respiratory distress in the preterm \& term neonate. Paediatrics 2008; 121(2): 419-32.

Sujeewa Amarasena

Professor, Department of Paediatrics, Faculty of Medicine, Ruhuna University 